\title{
Heat Deterioration of Phospholipids. III. Thermally Deteriorated Products from Phosphatidylethanolamine and Hexose
}

\author{
Nobutoshi Hamaguchi ${ }^{1}{ }^{*}$, Yoshihito Kasahara ${ }^{2}$, Akihito Hayashi, \\ Ryoji Sono ${ }^{1}$, Shin-ichi Tebayashi ${ }^{2}$, Chul-Sa KIM ${ }^{2}$ and Hen-Sik KoH ${ }^{1}$ \\ ${ }^{1}$ Research and Development Section, Tsuji Oil Mill Co., Ltd. \\ (565-1, Ureshinoniwanosho-cho, Matsusaka 515-2314, JAPAN) \\ ${ }^{2}$ Department of Bioresources Science, Faculty of Agriculture, Kochi University
}

(B200, Monobe, Nankoku 783-8502, JAPAN)

Accepted June 5, 2006 (received for review April 12, 2006)

\begin{abstract}
Novel four 2,3-dihydro- $1 H$-imidazo[1,2-a]pyridine-4-ylium derivatives were obtained with increase of UV absorption at $350 \mathrm{~nm}$ and browning of the solution by heating paste lecithin from soybean (SL) in octane. These four derivatives were characterized the factor based on UV absorption at $350 \mathrm{~nm}$. The SL was separated into the hexane (DSSL fr.) and 60\% ethanol in water frs. by liquid- liquid partition method. Even when the DSSL fr. was heated in octane more than $9 \mathrm{~h}$, the four derivatives were not formed and increase of any UV absorption was also not observed but the solution color was slightly yellowing. On the other hand, the formation of four derivatives and increase of UV absorption at $350 \mathrm{~nm}$ were observed when DSSL and $60 \%$ ethanol in water frs. were combined and, then, heated in octane. From the $60 \%$ ethanol in water fr, sucrose, raffinose and stachyose were isolated and identified as compounds which involved the formation of the derivatives with the DSSL fr. When DSPE (1,2-di-Ostearoyl-sn-glycero-3-phosphatidylethanolamine) and any hexose were combined and then heated, two 2,3-dihydro- $1 H$-imidazo[1,2-a]pyridine-4-ylium derivatives generated with increase of UV absorption at $350 \mathrm{~nm}$ and browning. From these results, it is concluded that the formation of derivatives, with increase of the UV absorption at $350 \mathrm{~nm}$ and browning, are based on the reaction of sugars and phosphatidylethanolamine (PE) in SL.
\end{abstract}

Key words: soybean lecithin, phospholipid, heat decomposition, phosphatidylethanolamine, 2,3-dihydro-1 $H$-imidazo[1,2-a]pyridine-4-ylium derivatives

\section{Introduction}

Recently, lecithin is not only used as an emulsifier, but also in various fields of application. Lecithin is improved into various forms to fit to use and application. However, these improvement processes sometimes cause its quality to lower, especially the improvement process via heating is known to make lecithin brown lowering its quality.
There are many reports for the browning and change of UV absorption of lecithin by heating. For example, Tomioka et al. (1-3) investigated that the browning of lecithin by heating involved polymerization of phosphatidylcholine (PC) and conjugated dienone compounds. Reaction of carbonyl compounds and PE browns lecithin, and forms pyridinium compounds with $\mathrm{UV}$ absorption at $280 \mathrm{~nm}(4,5)$. Increase of UV absorption at $240 \mathrm{~nm}$ in lecithin by heating is based on

\footnotetext{
*Correspondence to: Nobutoshi HamaguchI, Tsuji Oil Mill Co., Ltd., 565-1, Ureshinoniwanosho-cho, Matsusaka 515-2314, JAPAN

E-mail: n.hamaguchi@tsuji-seiyu.co.jp
} 
the carbonyl compounds (6). However, there is no report about browning and change of UV absorption of lecithin by heating in detail and the browning mechanism of lecithin by heating has been not known yet.

We recently discovered the absorption bands appeared at $350 \mathrm{~nm}$ besides those of $240 \mathrm{~nm}$ and 280 $\mathrm{nm}$ during the heating process of paste lecithin from soybean $(\mathrm{SL})(7,8)$. Novel four 2,3-dihydro- $1 \mathrm{H}$-imidazo[1,2-a]pyridine-4-ylium derivatives, namely compound A: 1-[2'-(2",3"-dilinoleoyl-sn-glycero-1"-phosphoethyl)]-6-methyl-2,3-dihydro- $1 H$-imidazo[1,2a]pyridine-4-ylium, compound B: 1-[2'-(2"-linoleoyl*3"-palmitoyl*-sn-glycero-1"'-phosphoethyl)]-6-methyl2,3-dihydro- $1 H$-imidazo[1,2- $a$ ]pyridine-4-ylium, compound C: 1-[2'-(2",3"-dilinoleoyl-sn-glycero-1"-phosphoethyl)]-6-hydroxymethyl-2,3-dihydro-1H-imidazo[1,2-a]pyridine-4-ylium, compound D: 1-[2'-(2"linoleoyl*-3"-palmitoyl*-sn-glycero-1"-phosphoethyl)]-6-hydroxymethyl-2,3-dihydro- $1 H$-imidazo[1,2a]pyridine-4-ylium, were isolated and identified as the compounds involving the increase of the UV absorption at $350 \mathrm{~nm}$ of SL by heating in octane (* means interchangeable, shown in Fig. 5). Each of four derivatives has two molar of PE moiety in its structure. Compounds $\mathbf{A}$ and $\mathbf{B}$ have a methyl group at 6 position of the 2,3-dihydro- $1 H$-imidazo[1,2- $a]$ pyridine-4-ylium ring. Compounds $\mathbf{C}$ and $\mathbf{D}$ have a hydroxymethyl group at 6 position of the ring. Compounds $\mathbf{A}$ and $\mathbf{C}$ have two molar of linoleoyl moiety in PE part and compounds $\mathbf{B}$ and $\mathbf{D}$ have one molar of linoleoyl moiety and one molar of palmitoyl moiety in PE part. The structures of these four derivertives are very similar. It is, therefore, very useful to consider the formation mechanism of these four compounds for understanding thermal deterioration of lecithin. In this paper, we report about compounds involving the formation of the derivatives and the mechanism in SL.

\section{Experimental}

\section{$\mathbf{2} \cdot \mathbf{1}$ Instruments}

GC data were measured with a HP 6890 Series Gas Chromatograph instrument fitted with a fused silica column $\{$ HP-5 (crosslinked 5\% PH ME Siloxane, $0.25 \mu \mathrm{m}$, thickness, $25 \mathrm{~m} \times 0.2 \mathrm{~mm}$ i.d.) $\}$ and programmed $170^{\circ} \mathrm{C}$ with FID mode. GC-MS data were measured by a JEOL JMS600 instrument with EI mode. ${ }^{1} \mathrm{H}$ - and ${ }^{13} \mathrm{C}$ NMR data were recorded by a JEOL JNM-AL400
(400MHz) spectrometer in $\mathrm{CDCl}_{3}$ or $\mathrm{D}_{2} \mathrm{O}$ with TMS as an internal standard. Letters (br-)s, (br-)d, t, q, and $\mathrm{m}$ represent (broad)singlet, (broad)doublet, triplet, quartet, and multiplet, respectively, and coupling constants are given in Hz. IR data were measured by a SHMADZU FT-IR 4300 in the liquid membrane method. HPLC were carried out with a Shimadzu LC-10AD pump equipped with Shimadzu RID-10A and Shimadzu SPDM10A diode array detectors. LC-MS data were recorded with a Shimadzu LC-MS 2010 mass spectrometer when using a column (Inertsil ODS-3 $150 \mathrm{~mm} \times 4.6 \mathrm{~mm}$ i.d., GL Sciences Inc., Japan, flow rate: $1.0 \mathrm{~mL} / \mathrm{min}$ ) eluted with $50 \%$ methanol in water in the APCI-positive mode. UV data were measured by a Hitachi 200-20 Spectrophotometer. In a two dimensional TLC analysis, chloroform/methanol/7 molar of aqueous ammonia $(130 / 60 / 8)$ and chloroform/methanol/acetic acid/water $(170 / 25 / 25 / 6)$ were used as a developer, respectively (9).

\section{$\mathbf{2} \cdot \mathbf{2}$ Material}

Paste soybean lecithin (SL) including PC(29.3\%), $\operatorname{PE}(25.7 \%), \operatorname{PI}(15.2 \%)$ and $\mathrm{PA}(11.2 \%)$ was manufactured by Tsuji Oil Mill Co., Ltd. (Mie, Japan). 1,2-Di$O$-stearoyl-sn-glycero-3-phosphatidylethanolamine (DSPE) was purchased from SIGMA (Missouri, USA). D-Galactose, D-glucose, D-fructose, sucrose, D-raffinose pentahydrate, stachyose tetrahydrate, Wakogel C-100 type and 5\% hydrochloric acid in methanol solution were purchased from Wako (Osaka, Japan). Sep-Pak C18 cartridge was purchased from Waters (Massachusetts, USA).

\section{$2 \cdot 3$ Extraction and Isolation}

\section{$2 \cdot 3 \cdot 1 \quad$ SL separation}

SL $(20.0 \mathrm{~g})$ was separated into hexane (DSSL, 250 $\mathrm{mL})$ and $60 \%$ ethanol in water fr. $(250 \mathrm{~mL} \times 4)$ by liquid-liquid partition, respectively. After drying the $60 \%$ ethanol fr. under reduced pressure, the fraction was applied on an ODS open column and successively eluted with water, $40 \%$ methanol in water and methanol.

$2 \cdot 3 \cdot 2$ SL, DSSL, DSPE heating method

SL (100 g) and DSSL (100 g of equivalent to SL) was dissolved in flasks with octane $(10 \mathrm{~L})$ and heated for $9 \mathrm{~h}$, respectively. After adding same equivalent of separated fractions or authentic sugars $(2 \mathrm{mmol})$ in small amount of aqueous methanol solution to the DSSL (20 g of equivalent to SL) in octane solution, the solvent was removed from octane. The DSSL octane 
solution $(250 \mathrm{~mL})$ was then heated for $9 \mathrm{~h}$. A mixture of DSPE $(480 \mathrm{mg}, 642 \mu \mathrm{mol})$ and galactose $(115 \mathrm{mg}$, $642 \mu \mathrm{mol})$ was also dissolved into octane $(100 \mathrm{~mL})$ and heated for $9 \mathrm{~h}$.

$2 \cdot 3 \cdot 3$ Isolation of compounds $\mathbf{1}$ and 2

After heating for $9 \mathrm{~h}$, the reactant of DSPE and galactose was chromatographed on a silica gel column (Wakogel C-100, $150 \mathrm{~mm} \times 10 \mathrm{~mm}$ i.d.), eluting in sequence with an increasing concentration of methanol in chloroform to obtain the chloroform, $25 \%$ methanol in chloroform, and 50\% methanol in chloroform frs. The two compounds ( 1 and $\mathbf{2} R t=26.9 \mathrm{~min}$ and 36.0 min, respectively) were isolated from the $50 \%$ methanol in chloroform fr. by using HPLC (column: Senshu Pak. AQUASIL SS-4251(60) $250 \mathrm{~mm} \times 10 \mathrm{~mm}$ i.d, Senshu, Japan, flow rate: $3.0 \mathrm{~mL} / \mathrm{min}$, solvent: chloroform: methanol: water $=70: 30: 1$, Detector: $U V$ at $350 \mathrm{~nm}$ ). The yields of compounds $\mathbf{1}$ and $\mathbf{2}$ were $16.45 \mathrm{mg} / \mathrm{g}$ and $6.46 \mathrm{mg} / \mathrm{g}$ based on a weight of DSPE and galactose, respectively.

\subsubsection{Methanolysis of compounds $\mathbf{1}$ and $\mathbf{2}$}

Compounds 1 ( $7.9 \mathrm{mg})$ or $\mathbf{2}(3.1 \mathrm{mg})$ was mixed with $1 \mathrm{~mL}$ of $5 \%$ hydrochloric acid in methanol, and heated at $85^{\circ} \mathrm{C}$ for $3 \mathrm{~h}$. After vacuum concentration, the reaction mixture was dissolved in $5 \mathrm{~mL}$ of ether and extracted four times with $4 \mathrm{~mL}$ of water. From the ether fr., methyl stearate was identified by using GC instrument and GC-MS \{ (programmed from $170^{\circ} \mathrm{C}$ ( 3 min hold) to $250^{\circ} \mathrm{C}$ at a rate of $10^{\circ} \mathrm{C} / \mathrm{min}$, measured at $\left.\left.70 \mathrm{eV}\right)\right\}$.

On the other hand, the water fr. was passed through a Sep-Pak C18 cartridge and successively eluted in sequence with an increasing concentration of methanol in water to obtain the water and $20 \%$ methanol frs. Compounds $\mathbf{A M}$ and $\mathbf{C M}$ were recovered from the water eluate. Compounds $\mathbf{A M}$ and $\mathbf{C M}$ were isolated by HPLC (column: Senshu Pak. AQUASIL SS4251(60) $250 \mathrm{~mm} \times 10 \mathrm{~mm}$ i.d, Senshu, Japan, flow rate: $3.0 \mathrm{~mL} / \mathrm{min}$, solvent: chloroform: methanol: water = 70: 30: 1, Detector: UV at $350 \mathrm{~nm}$ ). The spectral data of compounds 1, 2, AM and $\mathbf{C M}$ were given as follows.

\section{Compound 1}

${ }^{1} \mathrm{H}-\mathrm{NMR}\left(\mathrm{CDCl}_{3}\right) \delta: 0.88(6 \mathrm{H}, \mathrm{m}), 1.25$ (about $\left.50 \mathrm{H}, \mathrm{m}\right)$, $1.56(4 \mathrm{H}, \mathrm{m}), 2.26(4 \mathrm{H}, \mathrm{m}), 2.38(3 \mathrm{H}, \mathrm{s}), 4.10\left({ }^{*} \mathrm{H}, \mathrm{m}\right)$, $4.12\left({ }^{*} \mathrm{H}, \mathrm{m}\right), 4.23\left({ }^{*} \mathrm{H}, \mathrm{m}\right), 4.38\left({ }^{*} \mathrm{H}, \mathrm{m}\right), 5.16(1 \mathrm{H}, \mathrm{br}-$ s), $6.26(1 \mathrm{H}, \mathrm{br}-\mathrm{s}), 7.27(1 \mathrm{H}, \mathrm{br}-\mathrm{s}), 8.65(1 \mathrm{H}, \mathrm{br}-\mathrm{s})$.

* means not clear.

IR $v_{\text {MAX }}$ (liquid film) $\mathrm{cm}^{-1}: 1737(\mathrm{C}=\mathrm{O}), 1236(\mathrm{P}=\mathrm{O})$,
1060 (P-O).

Compound 2

${ }^{1} \mathrm{H}-\mathrm{NMR}\left(\mathrm{CDCl}_{3}\right) \delta: 0.89(6 \mathrm{H}, m), 1.29$ (about $\left.50 \mathrm{H}, \mathrm{m}\right)$, $1.56(4 \mathrm{H}, \mathrm{m}), 2.26(4 \mathrm{H}, \mathrm{m}), 3.93\left({ }^{*} \mathrm{H}, \mathrm{m}\right), 4.11\left({ }^{*} \mathrm{H}, \mathrm{m}\right)$, $4.34\left({ }^{*} \mathrm{H}, \mathrm{m}\right), 4.51\left({ }^{*} \mathrm{H}, \mathrm{m}\right), 5.18(1 \mathrm{H}, \mathrm{br}-\mathrm{s}), 6.40(1 \mathrm{H}$, br-s), 7.00 (1H, br-s), 8.60 (1H, br-s).

* means not clear.

Compound AM

$R t=18.2 \min . \lambda \max 351 \mathrm{~nm}$

LC-MS: $\mathrm{m} / \mathrm{z}(\%): 179 \mathrm{M}^{+}$

${ }^{1} \mathrm{H}-\mathrm{NMR}\left(\mathrm{D}_{2} \mathrm{O}\right) \delta: 2.23(3 \mathrm{H}, \mathrm{s}$, methyl), $3.77(4 \mathrm{H}, \mathrm{m}$, H-1' and H-2'), 3.89 (2H, t, $J=6.5, \mathrm{H}-3), 4.16(2 \mathrm{H}, \mathrm{t}, J$ $=6.5, \mathrm{H}-2), 6.26(1 \mathrm{H}, \mathrm{d}, J=4.3, \mathrm{H}-8), 7.10(1 \mathrm{H}, \mathrm{d}, J=$ 4.3, H-7), 8.14 (1H, s, H-5).

${ }^{13} \mathrm{C}-\mathrm{NMR}\left(\mathrm{D}_{2} \mathrm{O}\right) \delta: 11.90$ (q, methyl), 40.94 (t, C-2), 47.61(t, C-3), 58.74 (t, C-1'), 60.53 (t, C-2'), 116.44 (d, C-8), 123.12 (s, C-6), 127.88 (d, C-7), 147.82 (s, C-9), 150.68 (d, C-5).

\section{Compound $\mathbf{C M}$}

$R t=10.6 \mathrm{~min} . \lambda \max 346 \mathrm{~nm}$

LC-MS: $\mathrm{m} / \mathrm{z}(\%): 195 \mathrm{M}^{+}$

${ }^{1} \mathrm{H}-\mathrm{NMR}\left(\mathrm{D}_{2} \mathrm{O}\right) \delta: 3.84$ (4H, s, H-1' and H-2'), 3.99 (2H, br-t, H-2), $4.30(2 \mathrm{H}, \mathrm{t}, J=6.6$, hydroxymethyl), $4.62(2 \mathrm{H}, \mathrm{s}, \mathrm{H}-3), 6.45(1 \mathrm{H}, \mathrm{d}, J=4.4, \mathrm{H}-8), 7.16(1 \mathrm{H}$, br-d, H-7), 8.32 (1H, s, H-5)

${ }^{13} \mathrm{C}-\mathrm{NMR}\left(\mathrm{D}_{2} \mathrm{O}\right) \delta$ : 41.30 (t, hydroxymethyl), 47.94(t, C-2), 55.40 (t, C-3), 58.73 (t, C-1'), 60.99 (t, C-2'), 115.52 (d, C-8), 123.96 (s, C-6), 127.07 (d, C-7), 146.62 (s, C-9), 152.74 (d, C-5).

\section{Results and Discussion}

\subsection{UV Absorption Changes of SL and DSSL by Heating}

Heating SL in octane results in a change of its color from yellow to brown and gives an especially UV absorption at $350 \mathrm{~nm}$ with disappearance of PE in the solution on a two dimensional TLC analysis $(7,9)$. The color, was, however, slightly yellowing and any UV absorptions did not increase at all even when DSSL was heated in octane for more than $9 \mathrm{~h}$ in spite of this DSSL fr. contains the all lecithins from SL on the TLC analysis. Slight decrease of PE in the DSSL fr. was only observed on the TLC analysis. On the other hand, when the $60 \%$ ethanol in water fr., in which the lecithins 


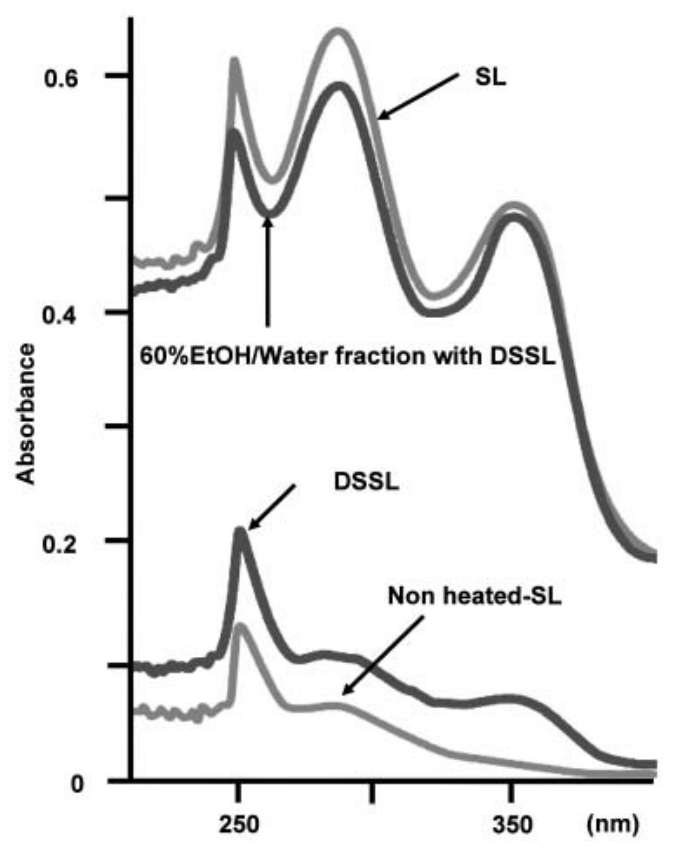

Fig. 1 UV Absorption Changes of SL and DSSL by Heating.

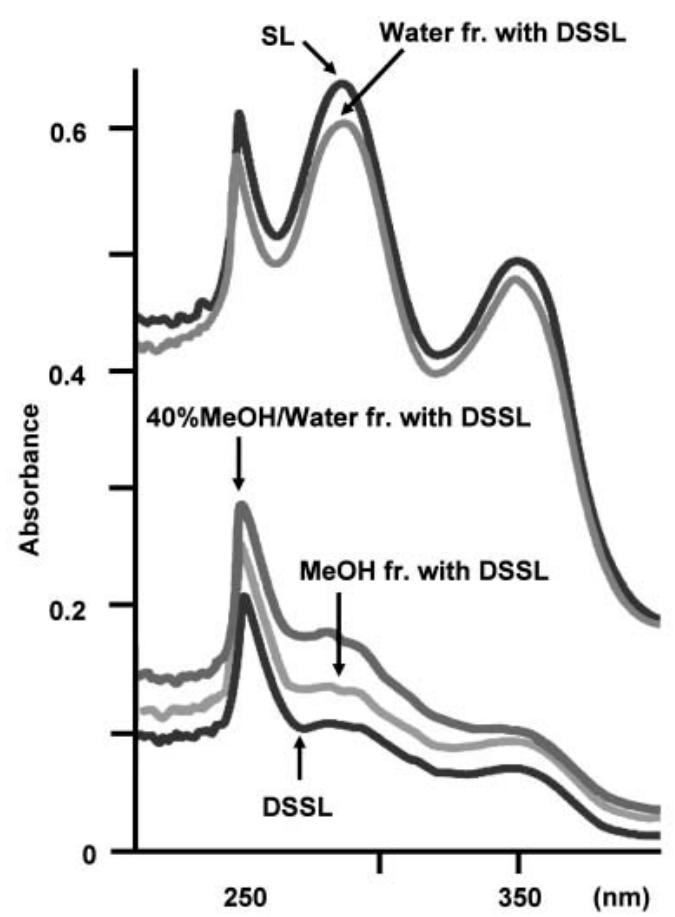

Fig. 2 UV Absorption Changes of DSSL by Heating with ODS Separated Frs. could not be detected on the TLC analysis, was added to DSSL and heated in octane, the color of reactant turned brown with increasing the UV absorption especially at $350 \mathrm{~nm}$ similar to that of SL (Fig. 1) and disappearance of PE in the solution was also observed on the TLC analysis. These results clearly show that the $60 \%$ ethanol in water fr. contains some compound(s) which are responsible for changing color and increasing especially UV absorption at $350 \mathrm{~nm}$ of the octane solution in cooperation with the DSSL. Of three fractions separated from the $60 \%$ ethanol in water fr. by using ODS open column (ODS-water, ODS-40\% methanol in water and ODS-MeOH frs), only the ODS-water fr. caused the resultant mixture to change color and to increase the UV absorption at $350 \mathrm{~nm}$ along with the DSSL (Fig. 2). The four 2,3-dihydro- $1 H$-imidazo[1,2-a]pyridine-4ylium derivatives (compounds A $\sim \mathbf{D}$ ) with UV absorption at $350 \mathrm{~nm}$ were also formed in the ODS-water fr (8). The other fractions (ODS-40\% methanol in water and ODS-methanol frs.) could not change the color, increase the UV absorption of the octane solution and form the four derivatives by heating. These results indicate that ODS-water fr. only contains some compound(s) which are responsible for changing color, increasing especially UV absorption at $350 \mathrm{~nm}$ of the octane solution and formation of the four compounds in cooperation with the DSSL.

\section{$3 \cdot 2$ Cooperation Function of Sugars with DSSL}

The components of the ODS-water fr. were further analyzed by using HPLC (column: Nakarai tesuque COSMOSIL $5 \mathrm{NH}_{2}$-MS, $250 \mathrm{~mm} \times 10 \mathrm{~mm}$ i.d., solvent: $75 \%$ acetonitrile in water, flow rate: $4.0 \mathrm{~mL} / \mathrm{min}$, detector: RI), Based on HPLC analysis, that the ODS-water fr. mainly composed of three compounds.

The structures of these three compounds were determined to be sucrose ( $12.69 \mathrm{mg} / \mathrm{g}$ of SL), raffinose (1.64 $\mathrm{mg} / \mathrm{g}$ of SL) and stachyose $(2.66 \mathrm{mg} / \mathrm{g}$ of SL) by comparing their HPLC chromatograms with those of authentic samples, respectively. These sugars are reported as typical sugars in soybean lecithin (10).

When the mixture of these sugars was added to the DSSL maintaining their ratio in the water fr. and heated under the same condition previously described, the resultant octane solution changed the color and increased the UV absorption at $350 \mathrm{~nm}$ like when SL was heated. The four 2,3-dihydro- $1 \mathrm{H}$-imidazo[1,2- 


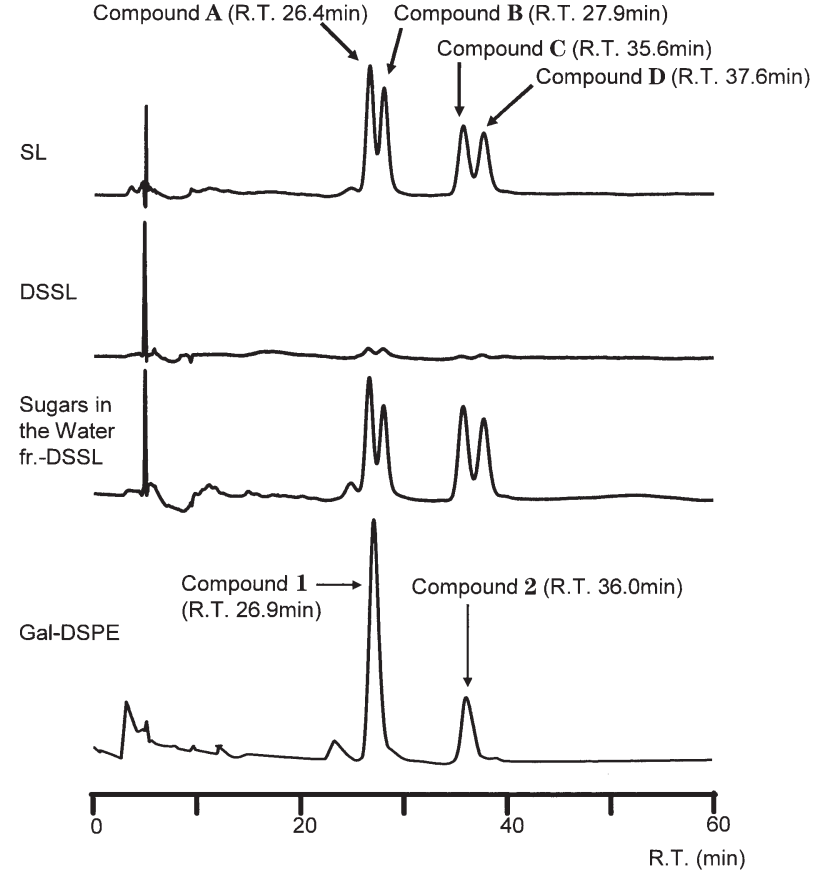

Fig. 3 HPLC Profiles of Compounds A D and Compounds $\mathbf{1}$ and $\mathbf{2}$.

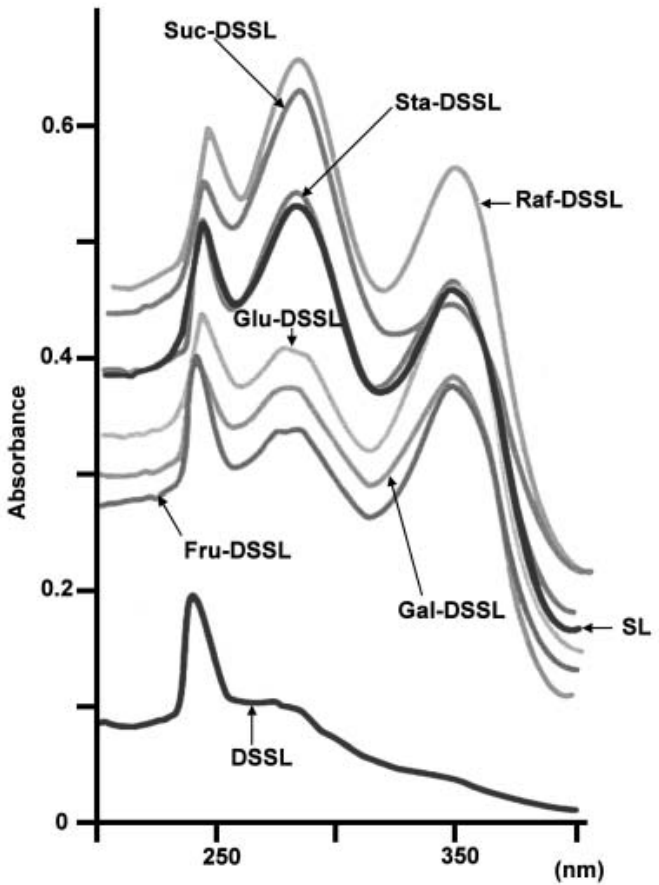

Fig. 4 UV Spectra of the Reactant of DSSL with Several Sugar.

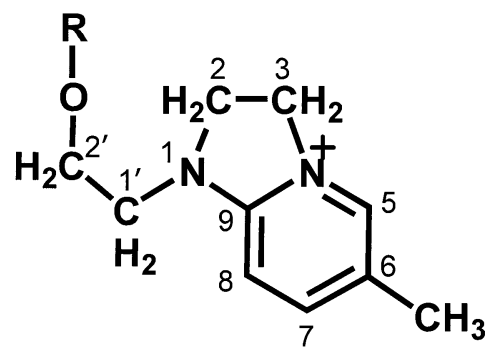

(1)

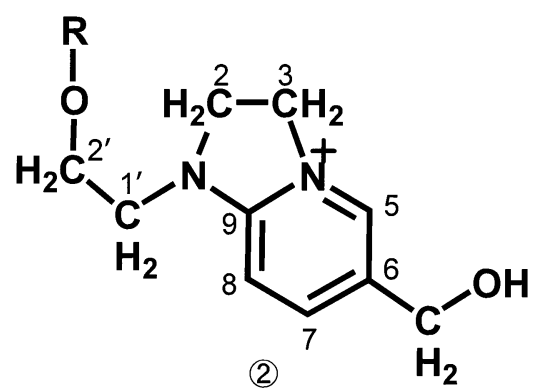

(2)

\begin{tabular}{|c|c|c|c|c|}
\hline \multirow{2}{*}{$\mathrm{R}=$} & \multicolumn{2}{|c|}{2 2",3"-diacyl-sn-glycero-1"-phospho moiety } & $\mathrm{H}$ \\
\hline Acyl groups & stearoyl & linoleoyl & linoleoyl and palmitoyl & \\
\hline (1) & Compound 1 & Compound A & Compound B & Compound AM* \\
\hline (2) & Compound 2 & Compound C & Compound D & Compound $\mathbf{C M}^{\star *}$ \\
\hline
\end{tabular}

* 6-methyl-2,3-dihydro-1H-imidazo[1,2-a]pyridine-4-ylium

** 6-hydroxymethyl-2,3-dihydro-1H-imidazo[1,2-a]pyridine-4-ylium

Fig. 5 The New Compounds with $\lambda$ max at $350 \mathrm{~nm}$ Isolated from Heated SL, DSPE with Hexose. 
a]pyridine-4-ylium derivatives (compounds A D) with UV absorption at $350 \mathrm{~nm}$ were also formed (Fig. 3).

From these results, we concluded that sugars in cooperation with the DSSL causes the typical UV absorption increase at $350 \mathrm{~nm}$ and color change from yellow to brown, due to formation of the compounds during heating.

\section{$3 \cdot 3$ UV Spectra of the Reactants of DSSL with Several Sugars}

To confirm the ability of cooperation function of sugars, several sugars, for example, galactose (Gal), glucose (Glu), fructose (Fru), sucrose (Suc), raffinose (Raf) and stachyose (Sta) were combined with DSSL and then heated, respectively. Even when any sugar were heated with DSSL, resultant octane solutions changed the color and increased UV absorption like when SL was heated as shown in Fig. 4 and four compounds (compounds $\mathbf{A} \sim \mathbf{D}$ ) with UV absorption at 350 nm were also formed (Fig. 5). From these results, we concluded that hexoses or oligosaccharides including a hexose moiety are based on making the color of DSSL solution brown and increasing the UV absorption at 350 $\mathrm{nm}$ by heating in octane.

Regardless a kind of hexoses including an aldose and a ketose, same results were obtained. These results might indicate that an oxygen atom on 2 position of sugar is concerned in the resultant reaction by heating and thus, it might be necessary to test 2-deoxysugars. A

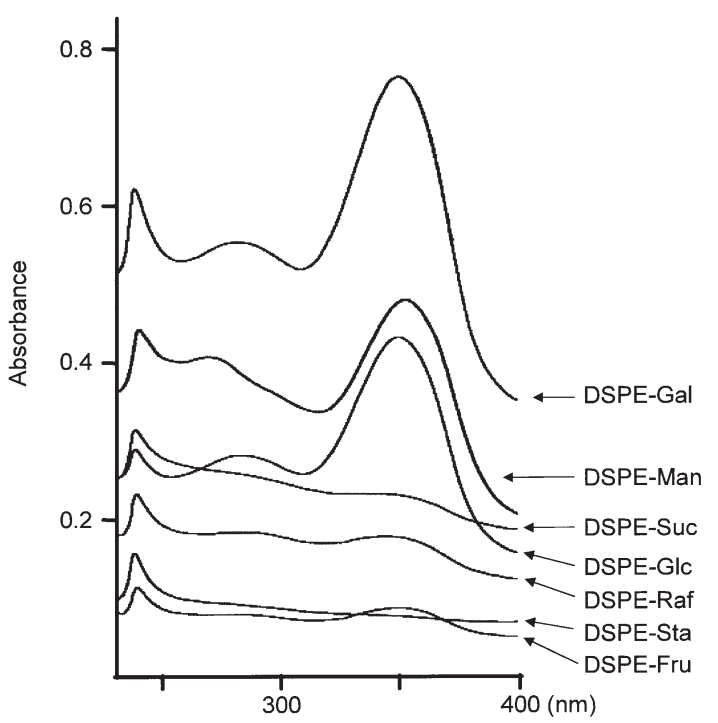

Fig. 6 UV Spectra of the Reactant of DSPE with Sugar. pentose, or heptose might be also investigated because only hexoses or oligosaccharides including a hexose moiety were used in this experiment.

\subsection{Heating Galactose with DSPE}

The compounds A D have two PE (phosphatidylethanolamine) moieties in the structure, respectively. Amount of PE in SL was decreased during heating in octane (9). These things may suggest that the decomposition of PE and formation of the four compounds occur simultaneously. To confirm the compound(s) being in cooperation with sugars in DSSL, DSPE (1,2-di-O-stearoyl-sn-glycero-3-phosphatidylethanolamine) and galactose were, therefore, heated in octane solution. When DSPE and galactose were heated in octane, increasing UV absorption at $350 \mathrm{~nm}$ and change of the color to brownish were observed in the reactant solution (Fig. 6). The four compounds A $\sim \mathbf{D}$ were not formed in the reactant solution but two compounds (compounds 1 and 2) with UV absorption at $350 \mathrm{~nm}$, which have similar retention times to those of compounds A D (Fig. 3), were formed. Even when various hexoses were used instead of galactose, the same compounds $\mathbf{1}$ and $\mathbf{2}$ were formed. These results clearly show that turning the color and increasing the UV absorption are based on the reaction a sugar with phosphatidylethanolamine.

Of these sugars, galactose made the UV absorption of the reactant highly increase. It is considered that this result is reasonable because open-chain form of galactose is most stable among hexoses tested, that is, aldehyde group in open-chain form of galactose could be attacked easier by nuculeophilic PE than that of the other hexoses.

\section{$3 \cdot 5$ Structural Determination of Compounds 1 and 2}

${ }^{1} \mathrm{H}$ - and ${ }^{13} \mathrm{C}-\mathrm{NMR}$ spectra of compounds $\mathbf{1}$ and $\mathbf{2}$ were very similar to those of compounds $\mathbf{A} \sim \mathbf{D}$ (8). All compounds contained a 2,3-dihydro- $1 H$-imidazo[1,2a]pyridine-4-ylium moiety in their structures, respectively. Structural difference between the compounds 1 and $\mathbf{2}$ are only substitute group on 6 position in the 2,3dihydro- $1 H$-imidazo[1,2-a]pyridine-4-ylium moiety, that is, 6 position in the ring of compound $\mathbf{1}$ is a methyl group, that of compound $\mathbf{2}$ is a hydroxymethyl group. There are not any other differences between the ${ }^{1} \mathrm{H}$ NMR data of compounds $\mathbf{1}$ and $\mathbf{2}$ except those of aro- 
matic ring. After conducting the methanolysis of compound $\mathbf{1}$, methyl stearate was only recovered from ether fr., while 1-(2'-hydroyethyl)-6-methyl-2,3-dihydro- $1 \mathrm{H}$ imidazo[1,2-a]pyridine-4-ylium, that is, compound AM which we previously reported, was obtained from water fr (8). On the other hand, 1-(2'-hydroxyethyl)-6hydroxymethyl-2,3-dihydro- $1 H$-imidazo[1,2-a]pyridine-4-ylium, that is compound $\mathbf{C M}$, was obtained by methanolysis of compound $\mathbf{2}$ from water fr (8).

According to these results, compounds $\mathbf{1}$ and $\mathbf{2}$ were determined as a 1-[2'-(2",3"-distearoyl-sn-glycero-1"'phosphoethyl)]-6-methyl-2,3-dihydro- $1 H$-imidazo[1,2a]pyridine-4-ylium and 1-[2'-(2",3"-distearoyl-sn-glycero-1"-phosphoethyl)]-6-hydroxymethyl-2,3-dihydro$1 H$-imidazo[1,2-a]pyridine-4-ylium, respectively (Fig. 5). The difference of retention times on HPLC between the compounds $\mathbf{A} \sim \mathbf{D}$ and compounds $\mathbf{1}$ and $\mathbf{2}$ is based on the difference of fatty acid moieties in their structures.

Change of the color from colorless to brown, increase of the UV absorption at $350 \mathrm{~nm}$, and formation of the compounds are caused at the same time even when only the DSPE and galactose were heated in octane. Formation mechanism of all six compounds is, therefore, same and each of six compounds is generated by reaction of two molar of PE and one molar of sugar during heating. It is concluded that reaction of PE with sugars is based on the deterioration in quality of SL by heating and it is very useful to remove the PE from SL to prevent SL from deteriorating in quality.

PE reacts with any hexose, regardless an aldose or a ketose, in octane solution by heating to give the same 2,3-dihydro- $1 H$-imidazo[1,2-a]pyridine-4-ylium derivatives. It will be, therefore, very important to investigate this reaction mechanism and identify of origin of car- bons in the derivatives.

\section{References}

1. F. TOMIOKA and T. KANEDA, Studies on the Brown Discoloration of Heated Phospholipids. I. The Browning Reaction of Lecithin. (1), Yukagaku, Vol. 23, 777-781 (1974).

2. F. TOMIOKA and T. KANEDA, Studies on the Brown Discoloration of Heated Phospholipids. II. The Browning Reaction of Lecithin. (2), Yukagaku, Vol. 23, 782-786 (1974).

3. F. TOMIOKA and T. KANEDA, Studies on the Brown Discoloration of Heated Phospholipids. III. The Browning Reaction of Lecithin. (3), Yukagaku, Vol. 25, 784-788 (1976).

4. T. NAKANISHI and K. SUYAMA, The Aldol Condensation Reaction of n-Saturated Aldehyde in the Presence of Phosphatidyl Ethanolamine, Nippon Nogei Kagaku Kaishi, Vol. 47, 313-319 (1973).

5. T. NAKANISHI and K. SUYAMA, Formation of Phosphatidyl 1-(2-Hydroxyethyl)-2,3,5-Trialkyl Pyridinium on Heating Phosphatidyl Ethanolamine with $n$-Alkanal, Agr. Biol. Chem., Vol. 38, 1141-1147 (1974).

6. C.H. LEA, Deteriorative Reactions Involving Phospholipides and Lipoproteins, J. Sci. Food Agr., Vol. 8, 1-13 (1957).

7. R. SONO, N. BAN, S. SAKAMOTO, N. HAMAGUCHI, S. TEBAYASHI, C.-S. KIM, H.-S. KOH and M. HORIIKE, Heat Deterioration of Phospholipids. I. Decomposition of Soybean Lecithin and Formation of New Products by Heating, J. Oleo Sci., Vol. 50, 905-911 (2001).

8. R. SONO, N. BAN, S. SAKAMOTO, N. HAMAGUCHI, S. TEBAYASHI, C.-S. KIM, H.-S. KOH and M. HORIIKE, Heat Deterioration of Phospholipids. II. Isolation and Identification of New Thermally Deteriorated Products from Soybean Lecithin, $J$. Oleo Sci., Vol. 51, 191-202 (2002).

9. Standard Methods for the Analysis of Fats, Oils and Related Materials, (Japan Oil Chem. Soc. ed.), 4.3.3.1-1996 (1996).

10. K. IKUTA, Y. OHTANI, Y. ISOBE and H. HARADA, Quantitative Determination of Oligosaccharides in Commercial Soybean Lecithin Products by High Performance Liquid Chromatography, Nippon Shokuhin Kogyo Gakkaishi, Vol. 41, 515-518 (1994). 